\title{
Multi-locus phylogeny using topotype specimens sheds light on the systematics of Niviventer (Rodentia, Muridae) in China
}

\author{
Bin Zhang ${ }^{1,2+}$, Kai He ${ }^{1,3,9^{*}+}$, Tao Wan ${ }^{1}$, Peng Chen ${ }^{4}$, Guozheng Sun ${ }^{5}$, Shaoying Liu ${ }^{6}$, Truong Son Nguyen ${ }^{7}$,
} Liangkong Lin $^{8}$ and Xuelong Jiang ${ }^{1 *}$

\begin{abstract}
Background: Niviventer is a genus of white-bellied rats that are among the most common rodents in the IndoSundaic region. The taxonomy of the genus has undergone extensive revisions and remains controversial. The current phylogeny is unresolved and was developed primarily on the basis of mitochondrial genes. Identification is extremely difficult, and a large number of GenBank sequences seem to be problematic. We extensively sampled specimens of Niviventer in China and neighboring northern Vietnam, including topotypes of the most reported species $(n=6)$, subspecies $(n=8)$, and synonyms $(n=4)$. We estimated phylogenetic relationships on the basis of one mitochondrial and three nuclear genes, using concatenation and coalescent-based approaches. We also employed molecular species delimitation approaches to test the existence of cryptic and putative new species.
\end{abstract}

Results: Our phylogeny was finely resolved, especially for the $N$. confucianus-like species. Our data provided the first support for N. brahma and N. eha as sister species, an assignment that is congruent with their morphological similarities. Species delimitation analyses provided new insight into species diversity and systematics. Three geographic populations of $N$. confucianus and one of $N$. fulvescens were supported as genetically distinct in our species delimitation analyses, while three recognized species ( $N$. coninga, N. huang, and N. lotipes) were not strongly supported as distinct.

Conclusions: Our results suggested that several genetically distinct species may be contained within the species currently known as N. confucianus and N. fulvescens. In addition, the results of Bayesian Phylogenetics and Phylogeography (BPP) for N. coninga, N. huang, and N. lotipes indicated that either inter-specific gene flow had occurred or imperfect taxonomy was present. Morphological examinations and morphometric analyses are warranted to examine the molecular results.

Keywords: Cryptic species, Species delimitation, Niviventer, Phylogenetics, Taxonomy, Topotype

\section{Background}

Taxonomy and systematics are crucial for understanding biological diversity and the conservation of species. Nonetheless, a complete understanding of species diversity remains a distant goal for most groups of organisms. A recent review of newly discovered mammal species showed that saturation of species discovery in Mammalia has not been reached. The newly added species

\footnotetext{
* Correspondence: hekai@mail.kiz.ac.cn; jiangxl@mail.kiz.ac.cn

†'Equal contributors

${ }^{1}$ State Key Laboratory of Genetic Resources and Evolution, Kunming Institute of Zoology, Chinese Academy of Sciences, Kunming, Yunnan, China

Full list of author information is available at the end of the article
}

include not only new described species, but also revisionary new species, which are synonyms or subspecies that have been recognized as valid species [1]. The latest version of Mammal Species of the World (2005) recognized 5,339 valid species, as well as 6,351 subspecies and 15,881 species-level synonyms [2]. A large proportion of synonyms and subspecies are assigned those ranks because of the lack of specimens, comprehensive analyses, and critical evaluation. This is especially the case for taxa that are widely distributed and morphologically unremarkable, such as the white-bellied rats of the genus

(c) The Author(s). 2016 Open Access This article is distributed under the terms of the Creative Commons Attribution 4.0 International License (http://creativecommons.org/licenses/by/4.0/), which permits unrestricted use, distribution, and reproduction in any medium, provided you give appropriate credit to the original author(s) and the source, provide a link to the Creative Commons license, and indicate if changes were made. The Creative Commons Public Domain Dedication waiver (http://creativecommons.org/publicdomain/zero/1.0/) applies to the data made available in this article, unless otherwise stated. 
Niviventer. These murine rodents are small to mediumsized and are mainly distributed in China, southern Himalaya, and Southeast Asia in various habitats over a wide range of elevations [3, 4]. While most species of Niviventer have very limited distributions, a few species, such as $N$. cremoriventer, $N$. confucianus, and $N$. fulvescens, are widely spread across large geographic areas and various landscapes [5-7].

The taxonomy of Niviventer has a long and complicated history. Representatives of Niviventer were originally included in the genus Mus (1836-1911), after which they were placed in Epimys (1911-1916), and Rattus (1916-1981; Rattus = Epimys, Hollister [8]), successively . The genus was established by Musser [3], who included 15 species and divided the genus into the $N$. andersonidivision and $N$. niviventer-division. According to the taxonomy presented by Musser and Carleton in 2005 [9], 17 taxa had full species status, while another 65 were recognized as subspecies or synonyms. The widespread $N$. cremoriventer, $N$. confucianus, and $N$. fulvescens had 10,14 , and 26 subspecies and synonyms, respectively. Recent studies integrating karyotypic information, DNA sequences, and morphological characters recognized $N$. lotipes (a synonym of $N$. tenaster) [10], $N$. huang, and $N$. bukit (synonyms of $N$. fulvescens) as full species [11] but placed N. langbianis into Chiromyscus [12]. Molecular phylogenetic studies further found that the widely distributed species were paraphyletic or even polyphyletic (e.g., $N$. andersoni, N. confucianus, $N$. excelsior, and N. fulvescens) [13]. Despite the possibility of misidentification, the number of putative species always exceeded that of recognized species $[13,14]$, implying that species diversity was underestimated. Thus, it was reasonable to re-evaluate the large body of remaining subspecies/synonyms within this genus.

The complex distribution pattern, large number of subspecies/synonyms, and morphological similarities make taxonomic and systematic revision for the genus Niviventer extremely difficult. Many species are morphologically very similar to one another [3]. For example, geometric morphometric analyses in a previous study showed that two distinctive genetic lineages largely overlapped (i.e., N. confucianus and N. fulvescens; Fig. 6 in $\mathrm{Lu}$ et al. [14]). Examination of type specimens is always crucial for taxonomic revision and essential for reevaluating the synonyms. However, the type series for most species distributed in Asia are deposited in natural history museums in Europe and the United States. Thus, comprehensive genetic analyses using topotypes may uncover cryptic species diversity and help to determine key taxa that need to be diagnosed before the type series is examined. In addition to the existing taxonomic problems, the phylogeny of Niviventer remained largely obscure. Although several major clades and species groups have been recognized, the relationships among them were unresolved, and the phylogenetic positions of $N$. coninga and $N$. culturatus were contradictory $[11,15]$. More than 540 Niviventer specimens have been sequenced, but most of the previous studies were conducted using only mitochondrial cytochrome b $(C Y T B)$ and cytochrome $c$ oxidase $(C O I)$ genes $[11,13$, $15,16]$. In another previous study using both mitochondrial (CYT B, COI, and D-loop) and nuclear (IRBP) loci, the gene trees were conflicting, suggesting that inclusion of multiple nuclear genes is warranted [14].

According to the taxonomy of Musser and Carleton [9] and recent studies [10-12], there are 10 recognized Niviventer species distributed in China, accounting for $55 \%$ of Niviventer species, including 5 endemic to China ( $N$. andersoni, $N$. coninga, $N$. culturatus, $N$. excelsior, and $N$. lotipes) and 5 both inside and outside China ( $N$. brahma, $N$. eha, $N$. confucianus, $N$. fulvescens, and $N$. huang). Nine Niviventer species ( $N$. cremoriventer, $N$. cameroni, $N$. rapit, $N$. fraternus, $N$. lepturus, $N$. hinpoon, $N$. tenaster, $N$. bukit, and $N$. niviventer) are distributed in Southeast Asia outside China. Another 24 taxa are recognized as synonyms or subspecies of $N$. andersoni $(n=3), N$. confucianus $(n=14), N$. eha $(n=1), N$. excelsior $(n=1), N$. fulvescens $(n=1)$, and $N$. huang $(n=4)$. Moreover, according to $\mathrm{Lu}$ et al. [14], undescribed species may exist in the mountains of southwestern China, an area characterized by extremely complex topography [17].

In the present study, we extensively sampled whitebellied rats throughout China and neighboring northern Vietnam, capturing topotypes of 18 species, subspecies, and synonyms. We sequenced four unlinked loci, reconstructed phylogenetic relationships, and delimited species boundaries. The goals were i) to clarify the status of species, subspecies, and synonyms, and ii) to uncover underestimated species diversity.

\section{Results}

\section{Genetic sequences}

A total of 157 Niviventer individuals representing 10 species were collected from China and neighboring northern Vietnam (Fig. 1, Table 1, Additional file 1: Table S1). We obtained 4,278 bp of sequences from 147 specimens, including 1,140 bp of mitochondrial DNA (mtDNA; CYT B [1,140 bp]) and 3,138 bp of nuclear genes (GHR [768 bp], IRBP [1,149 bp], and RAG1 $[1,221 \mathrm{bp}])$. The other ten individuals each failed to amplify one of the three nuclear genes. The new sequences have been deposited in GenBank under the accession numbers KY068361 to KY069019 (Additional file 1: Table S1). The appearance of premature stop codons or frame-shift mutations was not observed in coding regions. In addition, 537 sequences (517 CYT B, 


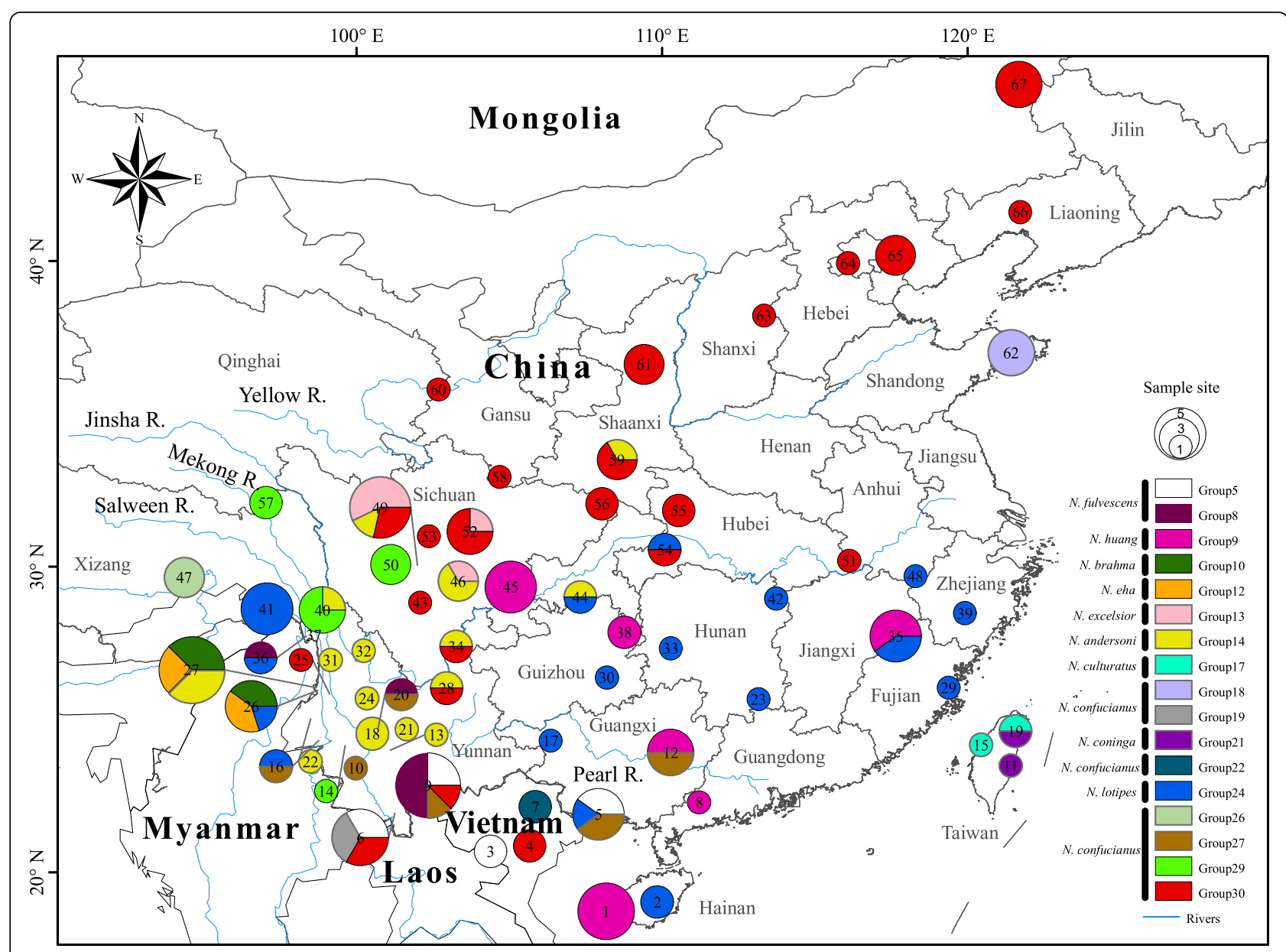

Fig. 1 Sample localities of specimens used in the present study. The numbers correspond to the locality numbers in Additional file 1: Table S1

$8 \mathrm{GHR}$, and 12 IRBP) representing 517 individuals of 17 Niviventer spp. (including $N$. andersoni, N. brahma, $N$. confucianus, $N$. coninga, $N$. culturatus, $N$. eha, $N$. excelsior, $N$. fulvescens, $N$. huang, $N$. bukit, $N$. hinpoon, $N$. niviventer, $N$. cremoriventer, $N$. tenaster, $N$. rapit, Niviventer sp. 1 and Niviventer sp. 2 [18]) were downloaded from GenBank (Additional file 2: Table S2). Sequences of Leopoldamys edwardsi, L. neilli, Rattus andamanensis, $R$. norvegicus, Chiromyscus chiropus, C. thomasi, $C$. langbianis, and Mus musculus were chosen as outgroups (Additional file 2: Table S2).

\section{Mitochondrial tree and species recovery}

The best-fit partitioning schemes and substitution models were determined by Partitionfinder V1.0.0 [19] under Bayesian Information Criterion (BIC) (Additional file 3: Table S3). The maximum likelihood (ML) tree calculated from CYT B sequences shows the 674 Niviventer individuals (Table 2) clustered into four major clades (i.e., clades A-D; Fig. 2), among which relationships were only weakly supported (bootstrap value, $\mathrm{BS} \leq 47$ ). Clade A was composed of $N$. fulvescens-like species (including $N$. bukit and N. huang) and three Southeast Asian species (N. rapit, N. hinpoon, and N. cremoriventer). Clade B was composed of two species from southwestern China and South Asia (N. eha and N. brahma). Clade C included $N$. andersoni and $N$. excelsior, representing the $N$. andersoni-division. All individuals in clade D were $N$. confucianus-like species, including subspecies and synonyms of $N$. confucianus, species previously included in $N$. confucianus (i.e., N. coninga, N. culturatus, and N. lotipes), and $N$. tenaster, which was morphologically similar to $N$. confucianus. In addition, individuals identified as N. bukit, $N$. eha, $N$. fulvescens, and N. niviventer in GenBank were recovered within two major clades each. The Kimura-2parameter (K2P) distances calculated in MEGA version 7 [20] between candidate species were 0.024 to 0.188 (Additional file 4: Table S4).

The species delimitation approach Automatic Barcode Gap Discover (ABGD) can partition sequences into groups or putative species based on gaps between intraand interspecific diversity of barcoded genes [21]. In this study, five ABGD analyses with different relative gap width values $(\mathrm{X}=0.5,1,1.5,2$, and 2.5$)$ were performed 
Table 1 The current taxonomy of the Niviventer genus distributed in China. All subspecies and synonyms are included. Taxa and topotypes included in the present study are shown. Sample locality numbers refer to those in Fig. 1 and Additional file 1: Table S1

\begin{tabular}{|c|c|c|c|c|}
\hline Status & Taxon & Type Locality & Included & $\begin{array}{l}\text { Topotypes } \\
\text { (sample locality number) }\end{array}$ \\
\hline Species & N. andersoni (Thomas, 1911) & Mt. Emei, Sichuan, China & Y & $Y(46)$ \\
\hline Subspecies & N. andersoni pianmaensis Li \& Yang, 2009 & Pianma, Yunnan, China & Y & $Y(27)$ \\
\hline Subspecies & N. andersoni ailaoshansis Li \& Yang, 2009 & Mt. Ailao, Yunnan, China & Y & $Y(13,18,21)$ \\
\hline Synonym & N. andersoni lushuiensis Wu \& Wang, 2002 & No specified & N & N \\
\hline Species & N. brahma (Thomas, 1914) & Mishmi Hills, Arunachal Pradesh & Y & $\mathrm{N}$ \\
\hline Species & N. confucianus (Milne-Edwards, 1871) & Baoxing, Sichuan, China & Y & $Y(52)$ \\
\hline Synonym & Rattus confucianus littoreus Cabrera, 1922 & Fuzhou, Fujian, China & $\mathrm{N}$ & $\mathrm{N}$ \\
\hline Synonym & Epimys zappeyi Allen, 1912 & Mt. Wa, Sichuan, China & N & $\mathrm{N}$ \\
\hline Synonym & Rattus confucianus yaoshanensis Shih, 1930 & Luoxiang, Guangxi, China & $\mathrm{N}$ & $\mathrm{N}$ \\
\hline Synonym & Rattus confucianus sinianus Shih, 1931 & Lechang, Guangdong, China & N & $\mathrm{N}$ \\
\hline Synonym & Rattus elegans Shih, 1931 & Lechang, Guangdong, China & $\mathrm{N}$ & $\mathrm{N}$ \\
\hline Subspecies & N. confucianus sacer (Thomas, 1908) & Yantai, Shandong, China & Y & $Y(62)$ \\
\hline Synonym & Mus confucianus luticolor Thomas, 1908 & Yan'an, Shaanxi, China & Y & $Y(61)$ \\
\hline Synonym & Epimys confucianus canorus Thomas, 1922 & Wenxian, Gansu, China & Y & $Y(58)$ \\
\hline subspecies & N. confucianus chihliensis (Thomas, 1917) & Eastern Tombs, Hebei, China & Y & $Y(65)$ \\
\hline subspecies & N. confucianus mentosus (Thomas, 1916) & Hkampti, Myanmar & Y & $\mathrm{N}$ \\
\hline subspecies & N. confucianus yushuensis (Wang \& Zheng, 1981) & Yushu, Qinghai, China & Y & $Y(57)$ \\
\hline subspecies & N. confucianus naoniuensis (Zhang \& Zhao, 1984) & Mt. Naoniu, Baicheng, Jilin, China & Y & $Y(67)$ \\
\hline subspecies & N. confucianus yajiangensis, Deng \& Wang, 2000 & Bajiaolou, Yajiang, Sichuan, China & Y & $Y(50)$ \\
\hline subspecies & N. confucianus deqinensis, Deng \& Wang, 2000 & Adong, Deqin, Yunnan, China & Y & $Y(40)$ \\
\hline Species & N. coninga (Swinhoe, 1864) & Taiwan, China & Y & $Y(11,19)$ \\
\hline Species & N. culturatus (Thomas, 1917) & Taiwan, China & Y & $Y(15,19)$ \\
\hline Species & N. eha (Wroughton, 1916) & Lachen, Sikkim, India & N & $\mathrm{N}$ \\
\hline Subspecies & N. eha ninus (Thomas, 1922) & Salween-Mekong divide, Yunnan, China & Y & $\mathrm{N}$ \\
\hline Species & N. excelsior (Thomas, 1911) & Kangding, Sichuan, China & Y & Y (49) \\
\hline Synonym & N. excelsior tengchongensis, Deng \& Wang, 2002 & No specified & $\mathrm{N}$ & $\mathrm{N}$ \\
\hline Species & N. fulvescens (Gray, 1847) & Katmandu, Nepal & Y & $\mathrm{N}$ \\
\hline Synonym & Rattus huang vulpicolor Allen, 1926 & Nating River, Yunnan, China & $\mathrm{N}$ & $\mathrm{N}$ \\
\hline Species & N. huang (Bonhote, 1905) & Guadun, Fujian, China & Y & Y (35) \\
\hline Synonym & Mus ling Bonhote, 1905 & Chungfengling, Fujian, China & Y & $Y\left({ }^{a}\right)$ \\
\hline Synonym & Rattus flavipilis Shih, 1930 & Luoxiang, Guangxi, China & Y & $Y(12)$ \\
\hline Synonym & Rattus flavipilis minor Shih, 1930 & Luoxiang, Guangxi, China & Y & $Y(12)$ \\
\hline Synonym & Rattus wongi Shih, 1931 & Lechang, Guangdong, China & N & $\mathrm{N}$ \\
\hline Species & N. lotipes (Allen, 1926) & Nada, Hainan, China & Y & $\mathrm{N}$ \\
\hline
\end{tabular}

${ }^{a}$ : The topotype of $N$. ling was sequenced in Lu et al. (2015) [14] and was included in the present study. Sample locality is not shown in Fig. 5

on 674 CYT B sequences, and two gaps (distance $=0.056$ and 0.121) were observed. Nevertheless, all analyses consistently supported a 30-group (i.e., candidate species) scenario only when intraspecific divergence $(p)=0.0121$ (Table 3; Fig. 2). In this scenario, 589 Chinese sequences were included in 22 groups (group 5, 8-19, 21-24, and 26-30; Additional file 1: Table S1 and Additional file 2: Table S2), and 157 sequences in the current study were included in 18 groups (excluding group 11 labeled $N$. eha eha, and including XZ-YD49 and XZ-YD50 from [14]; group 15 labeled $N$. andersoni, 04047 from [15]; group 16 labeled $N$. andersoni, 002 and 003 from [15]; and group 28 labeled $N$. eha, GLGS103 and GLGS104 from [15]; Additional file 1: Table S1). Two candidate species (groups 14 and 27) did not appear as monophyletic groups in our CYT B gene tree. Specimens identified as $N$. andersoni, $N$. bukit, $N$. 
Table 2 Sample sizes and species used for each analysis

\begin{tabular}{lllllllll}
\hline Data set & $\begin{array}{l}\text { Sample size } \\
\text { (from China) }\end{array}$ & $\begin{array}{l}\text { Species sampling } \\
\text { (from China) }\end{array}$ & CYTB & GHR & IRBP & RAG1 & Analyses & $\begin{array}{l}\text { Data source(s) } \\
\text { (current study + GenBank) }\end{array}$ \\
\hline Collected for this study & $157(153)$ & $10(10)$ & 157 & 154 & 154 & 152 & all & $/$ \\
Download from GenBank & $517(409)$ & $17(10)$ & 517 & 8 & 12 & $/$ & ML and ABGD & $/$ \\
CYT B data set & $674(562)$ & $18(10)$ & 674 & $/$ & $/$ & $/$ & ML and ABGD & $157+517$ \\
Concatenated data set & $204(176)$ & $18(10)$ & 204 & 162 & 166 & 152 & ML and MrBayes & $157+47$ \\
Full data set & $147(143)$ & $18(10)$ & 147 & 147 & 147 & 147 & BEAST, *BEAST, and BPP & $147+0$ \\
\hline
\end{tabular}

confucianus, $N$. cremoriventer, $N$. eha, $N$. excelsior, $N$. fulvescens, $N$. huang, and $N$. niviventer in GenBank were each found in at least two groups.

\section{Multilocus gene trees}

The phylogeny constructed from Bayesian and ML analyses of the mitochondrial-nuclear concatenated data (Table 2) resulted in very similar topologies, and only the MrBayes tree is shown (Fig. 3). Chiromyscus langbianis and $C$. chiropus were supported as sister species (posterior probabilities $[\mathrm{PP}]=1.0, \mathrm{BS}=94$ ), forming the sister clade to Niviventer ( $\mathrm{PP}=0.75, \mathrm{BS}=36$; Fig. 3 ). The four mitochondrial clades of Niviventer were also recovered. Compared to the CYT B gene tree, interspecific relationships were overall highly supported in clades $A$, $B$, and $C$, but remained unresolved in clade $D$ where only posterior probabilities are noted.

The phylogeny using 147 Niviventer sequences plus the outgroup (Fig. 4) was constructed according to the
mtDNA and concatenated data tree with better support for the relationships within the genus. In particular, the relationships among all clades and within clade D were better resolved in this phylogeny (Fig. 4).

\section{Species trees and species delimitation}

The species tree simulated in *BEAST showed similar topologies with the BEAST trees (Fig. 5), which assigned 147 individuals from the full data set (Table 2) into 18 groups according to the ABGD results. However, the sisterrelationship of groups 5 and 9 and the interspecific relationships in clade $\mathrm{D}$ were not robustly supported ( $\mathrm{PP}=$ 0.35-0.73). Furthermore, group 26 was strongly supported as a sister group to the other $N$. confucianus groups, excluding $N$. culturatus $(\mathrm{PP}=1)$. A sister relationship between groups 24 and 27 was strongly supported $(\mathrm{PP}=1)$, although their position was not stable $(\mathrm{PP}=0.72)$.

The BPP analyses based on the full data set provided similar results when different algorithms, priors, and

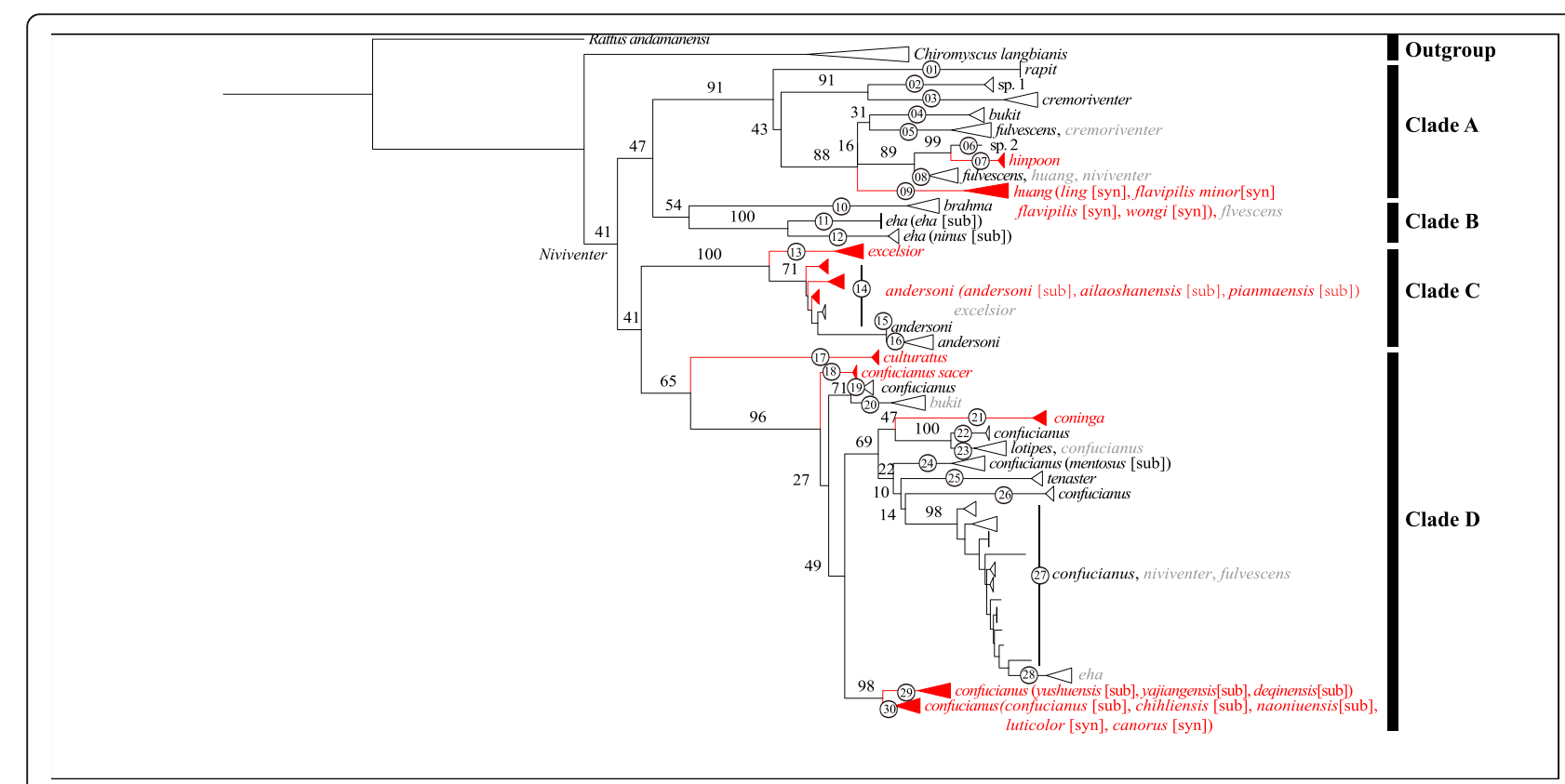

Fig. 2 Result of RAXML phylogenetic analyses of the CYT B gene. Node numbers indicate bootstrap values. Branch numbers represent putative species recognized by ABGD analyses. Clades in red color indicate topotypes included. Names after each clade are species names (subspecies names [sub] and synonym names [syn]) identified in this study and previous studies (sequences downloaded from NCBI GenBank). Names in black indicate irrefutable identification and in gray indicate likely misidentifications 
Table 3 Results of Automatic Barcode Gap Discovery (ABGD) analyses with $\mathrm{K} 80$ distance model

\begin{tabular}{lll}
\hline X (Gap width) & $p=0.0056$ & $p=0.0121$ \\
\hline 0.5 & 53 & 30 \\
1.0 & 48 & 30 \\
1.5 & 41 & 30 \\
2.0 & 41 & 30 \\
2.5 & 36 & 30 \\
\hline
\end{tabular}

The number of putative species are calculated based on different widths of gaps $(\mathrm{X})$, and priors of intraspecific divergence $(\mathrm{p})$

starting trees were used, and high effective sample sizes (ESSs) $(\geq 200)$ were observed for all parameters. We adopted a conservative criterion, considering $\mathrm{PP} \geq 0.95$ as strong support for a putative species (Table 4; Additional file 5: Table S5). When both mitochondrial and nuclear genes were used, 15 putative species were recognized (Fig. 5; Table 4; Additional file 5: Table S5); the nuclear genes alone supported an 11-species scenario (Fig. 5; Table 4; Additional file 5: Table S5). All BPP analyses consistently supported group 5 (from southern China and Southeast Asia, recognized as $N$. fulvescens, Additional file 6: Table S6) and group 9 (identified as $N$. huang) as the same species. Group 8 was supported as a distinct putative species (putative sp. 3). Animals in this group were from Tibet, Yunnan, China, and northern Vietnam, and were morphologically similar to $N$. fulvescens, but did not have distinctively bicolored tails [5] (Additional file 7: Table S7).

When nuclear genes alone were used, BPP supported $N$. coninga (group 21), N. lotipes (group 23) and several populations of $N$. confucianus as one species (Fig. 5; Table 4; Additional file 5: Table S5). All analyses supported group 24 from western Yunnan and southeastern Tibet (identified as $N$. confucianus mentosus); group 26 from Gongbu, Tibet (identified as N. confucianus; putative sp. 4); and group 27 from southern China and northern Vietnam (identified as $N$. confucianus; putative sp. 5) as three putative species (Fig. 5; Table 4; Additional file 5: Table S5).

\section{Discussion}

Correct naming of species

According to the famous Chinese teacher Confucius, "If names be not correct, language is not in accordance with the truth of things. If language be not in accordance with the truth of things, affairs cannot be carried on to success" [22]. The species $N$. confucianus was named after Confucius [23], and his quote regarding names is indeed relevant to the taxonomy of the genus Niviventer. Species diversity within this genus has been underestimated [13, 14], and our understanding of its taxonomy was incomplete [9]. These two issues have prevented a full understanding of the systematics and evolution of

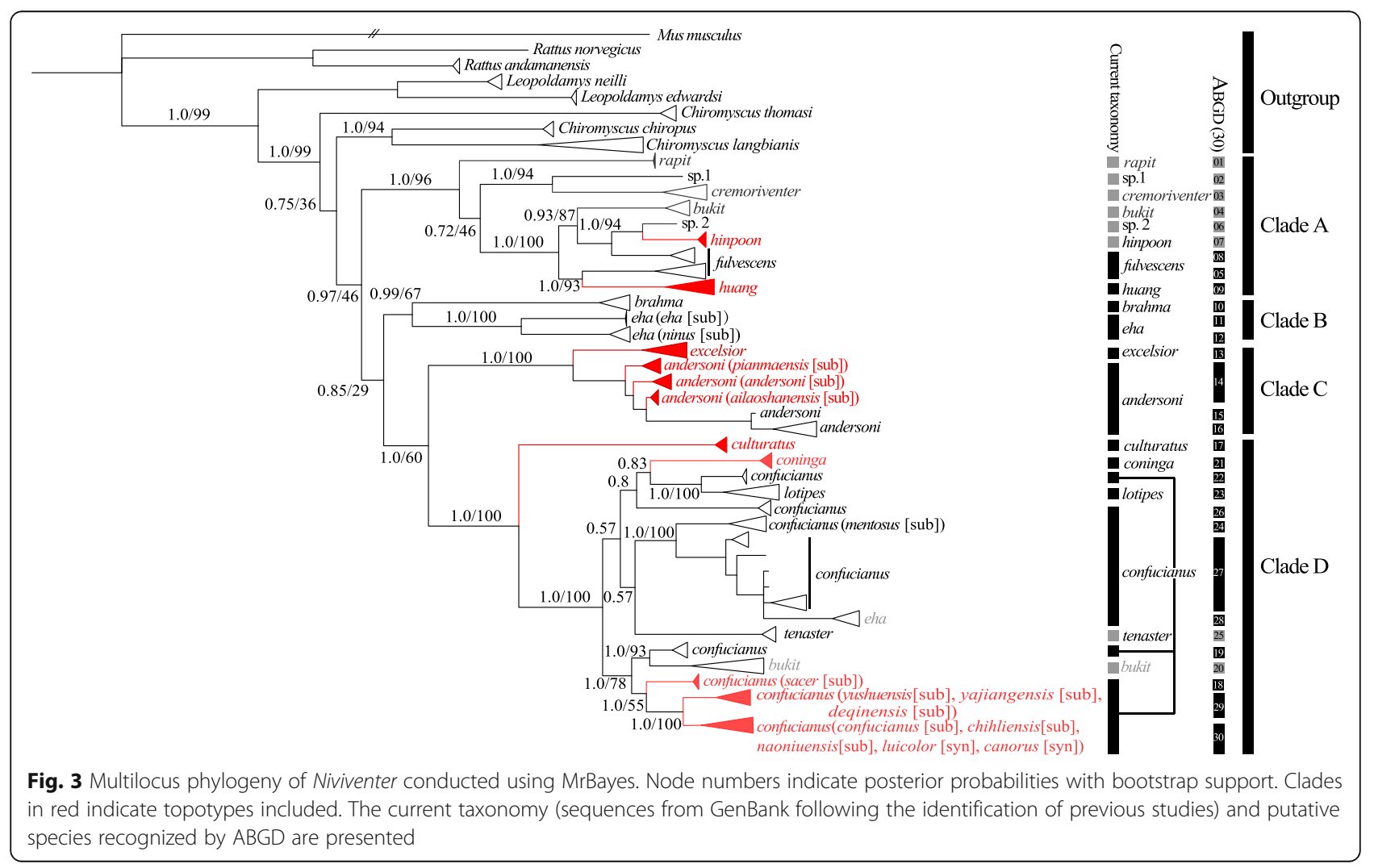




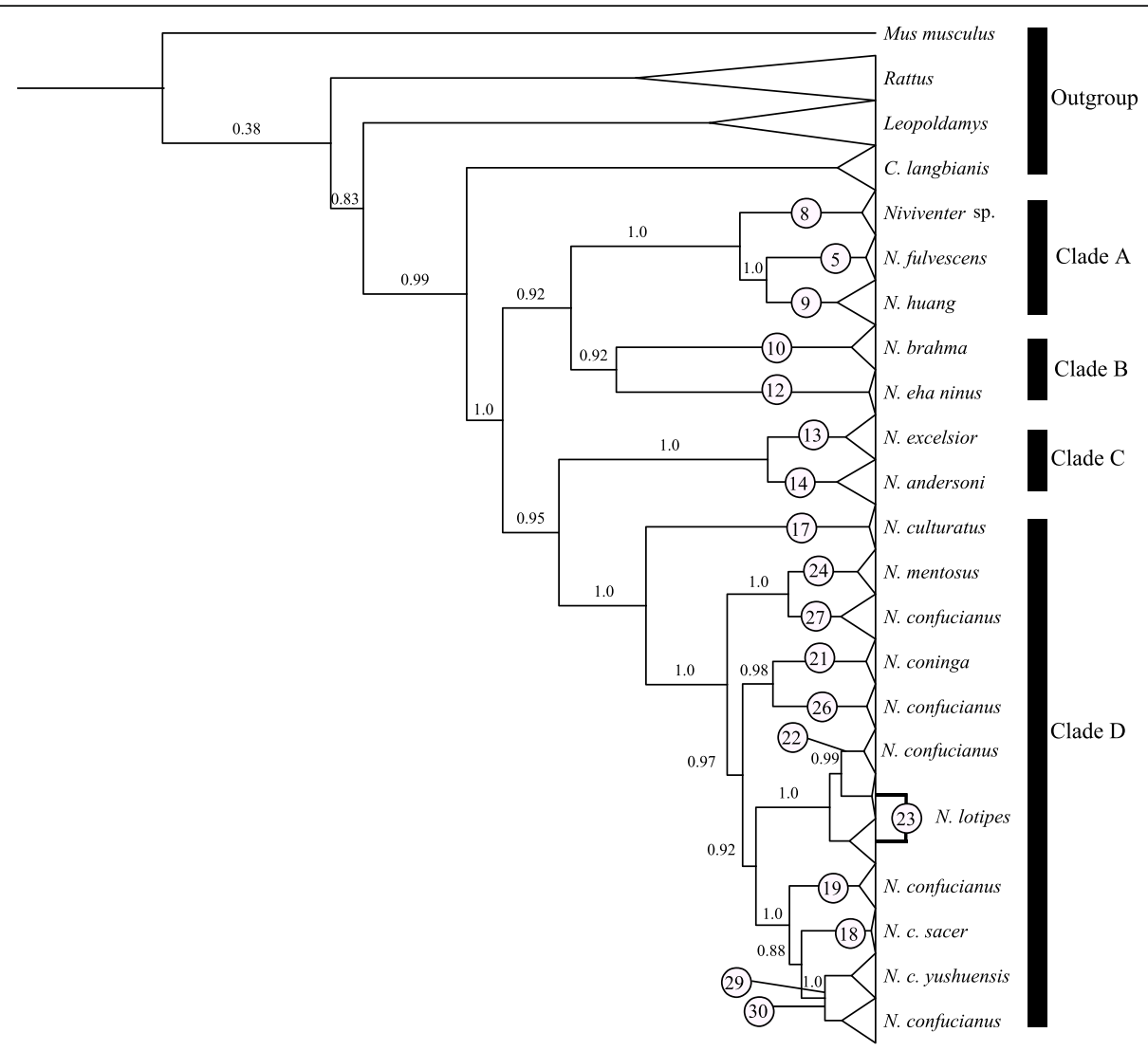

Fig. 4 Multilocus phylogenetic tree of full data set using BEAST. Numbers above the nodes indicate Bayesian posterior probabilities (BPP)

the genus. Multi-locus sequencing for topotype, conducted in this study, indicates the need for several taxonomic and systematic revisions.

Implications of the molecular species delimitation analyses Based on the barcoding gap found from the pairwise distances of $C Y T$ B sequences, the single gene species delimitation method, ABGD, supported the existence of 22 groups/putative species in China (represented by 589 sequences from China), in agreement with previous reports by He and Jiang [13] and Lu et al. [14]. Although far fewer samples $(n=147)$ were available for multilocus species delimitation, BPP analyses still recognized 15 (using nuclear plus mitochondrial genes) or 11 (using three nuclear genes alone) putative species.

BPP molecular species delimitation approaches have been developed and widely used in mammals [24, 25], amphibians [26, 27], and reptiles [28, 29]. It should be noted that the biological species concept associated with BPP may not be true in practice [30], and that simulations showed that BPP is robust in the case of incomplete lineage sorting but sensitive to hybridization. Strongly supported putative species could be either valid species or long-term isolated conspecific populations. On the other hand, when named species were not strongly supported $(\mathrm{PP}<0.95)$ as putative species by $\mathrm{BPP}$, the results either suggested genetic homogenization (i.e., conspecific relationships) or gene flow between valid species. In both cases, the taxonomic status must still be evaluated carefully in a taxonomic context and must not violate the International Code of Zoological Nomenclature [31]. The BPP analysis that used nuclear genes alone did not strongly support $N$. huang, $N$. lotipes, or $N$. coninga as putative species. On the other hand, four putative new species (N. confucianus mentosus and Niviventer sp. 3-5) need further attention. Comprehensive morphological and morphometric examinations are warranted to test species status for all these taxa.

\section{Value of topotypical samples}

Species in the genus Niviventer are morphologically very similar. The current taxonomy roughly relies on the size of skull, the length of ear and tail, and the color of skin and feet [3]. Morphological identification is not only time-consuming but also challenging. All but one of the topotypes of Niviventer sequenced in the current study were presented for the first time. We carefully assigned each individual to recognized species on the basis of morphology (Suppl. File 2) and the molecular results. These voucher specimens and sequences are valuable for 


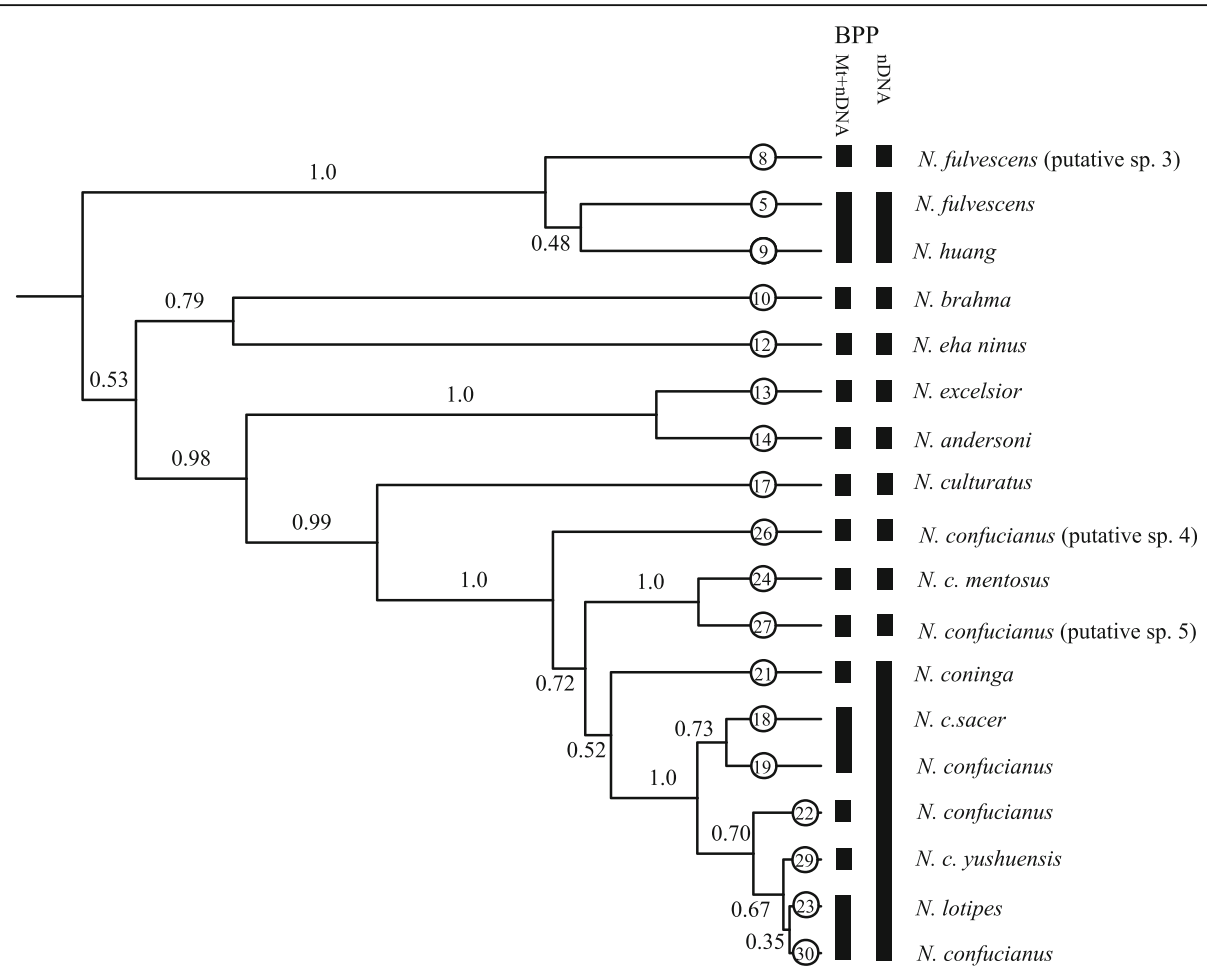

Fig. 5 A coalescent-based species tree referred using the *BEAST model based on the result of ABGD analyses. The putative species recognized by Bayesian Phylogenetics and Phylogeography (BPP) using mitochondrial and nuclear genes and using nuclear genes alone are presented. Node numbers indicate posterior probabilities estimated in *BEAST

barcoding-based species identification and taxonomic revision. As shown in our $C Y T B$ gene tree, GenBank sequences under the same species names were found in different groups or major clades (Fig. 1), indicating misidentification.

\section{Implications for taxonomy}

The analyses of topotype sequences of named synonyms and subspecies revealed multiple putative species within the currently recognized species of $N$. confucianus, $N$. fulvescens, N. andersoni, and N. eha. Within N. confucianus, the subspecies $N$. confucianus mentosus should be examined more closely, as it was supported as a distinct group/putative species in all analyses. This subspecies was named and described as a species by Thomas [32] on the basis of the holotype from Hkampti, Myanmar, after which it was assigned as a subspecies of $N$. niviventer [33] or a synonym of $N$. confucianus [3]. Animals from Chayu (Tibet Autonomous Region), and Nujiang (Yunnan Province) were assigned to $N$. c. mentosus on the basis of their longer hind feet $(\geq 30 \mathrm{~mm})$ and whitetipped tail [34]. Niviventer sp. 4 collected from Guangxi and Yunnan were marked by a white tail tip and conspicuous brush, a typical pattern of $N$. confucianus that distinguishes it from N. c. yaoshanensis, $N$. niviventer, or $N$. fulvescens. Niviventer sp. 3 is similar to N. fulvescens in body size and color pattern but could be distinguished from $N$. fulvescens and $N$. cremoriventer by its fuzzy bicolor tail.

Our analyses also suggest that diversity in some groups may be overestimated. Niviventer lotipes (group 23), recognized by Li et al. [10] for its distinct karyotype, was not

Table 4 Results of Bayesian phylogenetics and phylogeography (BPP) analyses using coalescent species trees as guard trees

\begin{tabular}{|c|c|c|c|c|c|c|c|c|c|c|c|}
\hline $\begin{array}{l}\text { Mitochondrial } \\
\text { phylogroups }\end{array}$ & 5,9 & $8,5+9$ & 10,12 & 13,14 & 24,27 & 23,30 & $29,23+30$ & $22,23+29+30$ & 18,19 & $\begin{array}{l}18+19,22+29+ \\
23+30\end{array}$ & $\begin{array}{l}21,18+19+22+29 \\
+23+30\end{array}$ \\
\hline $\begin{array}{l}\text { MtDNA and nuclear } \\
\text { Algorithm } 0\end{array}$ & 0.21 & 1.0 & 1.0 & 1.0 & 1.0 & 0.74 & 0.99 & 0.99 & 0.05 & 1.0 & 1.0 \\
\hline $\begin{array}{l}\text { MtDNA and nuclear } \\
\text { Algorithm } 1\end{array}$ & 0.21 & 1.0 & 1.0 & 1.0 & 1.0 & 0.74 & 0.99 & 1.0 & 0.05 & 1.0 & 1.0 \\
\hline nuclear Algorithm 0 & 0.15 & 1.0 & 0.99 & 1.0 & 1.0 & 0.08 & 0.20 & 0.31 & 0.07 & 0.55 & 0.71 \\
\hline nuclear Algorithm 1 & 0.19 & 1.0 & 0.99 & 1.0 & 1.0 & 0.08 & 0.26 & 0.32 & 0.05 & 0.52 & 0.65 \\
\hline
\end{tabular}


supported as a putative species by any of our BPP analyses. It was originally described as a subspecies of $N$. confucianus [35] on the basis of the holotype from Nada, Hainan, then treated as a subspecies of $N$. niviventer [33, 36] or a synonym of $N$. tenaster [9]. We did not included any topotype of $N$. lotipes (Danzhou, Hainan), but all specimens we included from Hainan, Fujian and Guangxi could be affiliated with the holotype (AMNH M-59303) of $N$. lotipes based on the sharp bicolor appearance of the tail, and are different from specimens used in Li et al. [10], showing a sharp bicolor appearance. However, individuals from other localities had a white-tipped tail. Another recognized species, N. coninga (group 21), was not supported by the BPP analyses using nuclear genes. Furthermore, most recognized subspecies of $N$. confucianus were clustered into two lineages or groups (subspecies N. c. yushuensis, N. c. yajiangensis, and N. c. deqinensis in group 29, and the nominated subspecies $N$. c. confucianus, $N$. c. chihliensis, and N. c. naoniuensis in group 30). They were not supported as putative species by BPP analyses. Therefore, the taxonomic status of all these clades must be reexamined.

\section{Conclusions}

In this study, we collected extensive samples of Niviventer in China, including most topotypes of species, subspecies, and synonyms. We developed a robust phylogeny on the basis of one mitochondrial and three nuclear genes. Four major clades were recovered by both concatenation and coalescent-based approaches. The sister relationship between $N$. brahma and $N$. eha was well supported, and relationships among $N$. confucianus-like species were mostly well resolved. Our coalescent-based species delimitation analyses indicated that species diversity of Niviventer may be overestimated by single locus in previous studies, and three species should be re-evaluated using morphological or morphometric approaches. However, our analyses also indicated that cryptic or putative new species may exist in the mountains of southwest China, an area characterized by extremely complex topography, climate conditions, and geographic history.

\section{Methods \\ Collection of samples and sequences}

We collected 157 individuals of Niviventer, as well as five Chiromyscus langbianis (previously included in Niviventer), from China and neighboring northern Vietnam (Additional file 1: Table S1). These samples were collected at 67 locations (Fig. 1), including the type localities of 6 species, 8 subspecies, and 4 synonyms (Table 1). Voucher specimens from mainland China were deposited in the Kunming Institute of Zoology, Chinese Academy of Sciences, and Sichuan Academy of Forestry, China, and voucher specimens from Taiwan were deposited in Tunghai University. Specimens were carefully identified on the basis of external and skull characters following the original descriptions (Additional file 6: Table S6, $[3,5,6])$ and in consideration of the results of molecular phylogenetic analyses (see Results section). We followed the taxonomy of Musser and Carleton [9] but recognized N. huang, N. bukit, and N. lotipes as valid species $[10,11]$, and treated $N$. langbianis as a member of Chiromyscus following Balakirev et al. [12]. After completing our mitochondrial phylogeny, we carefully examined and described the morphological characters of each clade (Additional file 7: Table S7).

Total genomic DNA was extracted using the sodium dodecyl sulfate (SDS) method [37]. The complete mitochondrial cytochrome $\mathrm{b}(C Y T B)$ gene and three nuclear gene segments (Exon 10 of the growth hormone receptor $[G H R]$, Inter-photoreceptor retinoid binding protein $[I R B P]$, and Recombination activation gene 1 [RAG1]) were amplified. Primers and annealing temperatures were either taken from the literature [38-40] or designed for this study (Table 5). PCR products were purified and sequenced in both directions using the BigDye Terminator Cycle kit v. 3.1 (Invitrogen, USA) on an ABI 3730xl sequencer (Applied Biosystems, USA).

Table 5 Primers and polymerase chain reaction (PCR) cycling conditions used in this study

\begin{tabular}{|c|c|c|c|c|c|}
\hline Genes & Primer Name & Nucleotide sequence $5^{\prime}$ to $3^{\prime}$ & Annealing Temperature $\left({ }^{\circ} \mathrm{C}\right)$ & Fragment Length (bp) & Citation \\
\hline \multirow[t]{2}{*}{ CYT B } & L14724_hk3 & GGACTTATGACATGAAAAATCATCGTTG & $47.5^{\circ} \mathrm{C}$ & 1140 & He et al. 2010 [38] \\
\hline & H15915_hk3 & TCTCCATTTCTGGTTIACAAGAC & & & \\
\hline \multirow[t]{2}{*}{ IRBP } & IRBP-217 & ATCCCCTATGTCATCTCCTACYTG & $60^{\circ} \mathrm{C}$ & 1149 & Stanhope et al. 1992 [39] \\
\hline & IRBP-1531 & CGCAGGTCCATGATGAGGTGCTCCGTGTCCTG & & & \\
\hline \multirow[t]{2}{*}{ RAG1 } & S70 & TCCGAGTGGAAATTTAAGMTGTT & $51.7^{\circ} \mathrm{C}$ & 1221 & Steppan et al. 2004 [40] \\
\hline & S73 & GAGGAAGGTRTTGACACGGATG & & & \\
\hline \multirow[t]{2}{*}{ GHR } & GHR-F & GAGTTCATTGAGCTGGATAT & $60^{\circ} \mathrm{C}$ & 777 & Current Study \\
\hline & GHR-R & ATGAGTTGCGCTGACGA & & & \\
\hline
\end{tabular}


We assembled and edited sequences using DNASTAR Lasergene v. 7.1 and aligned each gene using MUSCLE [41]. MEGA 7 was used for visual inspection [20].

\section{Phylogenetic reconstruction}

Three data sets were used for phylogenetic analyses: 1) the CYT B data set, which included 674 in-group sequences (157 sequences from the current study and 517 sequences downloaded from GenBank; Table 2), representing 16 of 19 recognized Niviventer species and two potential new species identified in a previous study (namely, Niviventer sp. 1 and sp. 2) [18], with Rattus andamanensis and Chiromyscus langbianis as the outgroup. The first $60 \mathrm{bp}$ and the last $63 \mathrm{bp}$ of $C Y T$ B were deleted from the data set to avoid sequencing errors [13]. 2) A four-gene concatenated data set, including sequences of 197 individuals of Niviventer plus outgroups (157 sequences from the current study and 40 sequences downloaded from NCBI GenBank; Tables 2). One to four sequences downloaded from GenBank were selected as representative of each group based on the CYT B gene tree. 3) The full data set, including 147 sequences from the current study plus 8 individuals representing six outgroup species (C. langbianis, $L$. edwardsi, $L$. neilli, $R$. andamanensis, $R$. norvegicus, and $M$. musculus), all of which have four gene segments (CYT B, GHR, IRBP, and RAG1).

PartitionFinder v. 1.0.0 [19] was used to estimate the best-fit partitioning schemes and substitution evolutionary models under the Bayesian Information Criterion $[42,43]$. For concatenated gene tree/species tree analyses, we concatenated gene alignments and defined by genes and codon positions. We did not consider the parameter for the proportion of invariant sites suggested by the author of RAxML [44]. Thus, we considered 12 models for each MrBayes and BEAST. For RAxML analyses, only GTR + gamma models were considered because RAxML did not allow any other models. For coalescent species tree estimation (see below), each of the three nuclear genes was treated as one partition and the CYT B was partitioned by codon positions. jModelTest 2 was used to estimate the best-fit evolutionary model for each nuclear gene [45].

The phylogenetic relationships of Niviventer were reconstructed using Bayesian inference and ML approaches. ML analyses and Bayesian analyses were conducted for the CYT B data set and the full data set, respectively, and both ML and Bayesian analyses were used for the concatenated data set. The ML analyses were performed using RAxML v7.2.8 on the CIPRES Science Gateway v 3.3 [46] (http://www.phylo.org). The BS were obtained using a rapid bootstrapping algorithm with 500 bootstrap replicates [44]. Bayesian analyses of the concatenated data set were implemented in MrBayes v. 3.2 and repeated twice [47]. Each analysis consisted of two independent runs, using four chains, sampled every 1,000 generations. We used Tracer v. 1.6 [48] to access the convergence of the two independent runs and stationary state of each parameter, and ESSs higher than 200 were considered adequate. Finally, each analysis was run for 2 million generations, and the first $25 \%$ of the generations were discarded as burn-in. Bayesian analyses of the full data set were implemented in BEAST v. 1.8.2 [48]. Each BEAST analysis used a lognormal relaxed clock model, a birth-death tree prior [49] and the other default parameters. The analyses were repeated twice; each was run for 200 million generations, and we sampled every 20,000 interactions. We considered $\mathrm{BS} \geq 70$ and $\mathrm{PP} \geq 0.95$ as strong support $[50,51]$.

\section{Species delimitation and species tree estimation}

We used ABGD [21] to recover candidate species, and species tree and Bayesian Phylogenetics and Phylogeography (BPP v. 3.1; [52]) for validation. The ABGD approach assumed that the largest intraspecific divergence is still less than the smallest interspecies divergence. The gap between intra- and interspecific distance observed from the distribution of sequence distances is defined as a barcoding gap. ABGD automatically clusters the sequences into groups or hypothetical species based on the barcoding gap. Thus, detection of pairwise differences is the premise of this method. The only input parameter, although imprecisely, was the maximum prior intraspecific distance, $p$. We chose ABGD instead of bPTP [53] or GMYC [54] because this method was independent of phylogenetic trees and particularly sensitive to recent speciation events [55]. This analysis was performed on the ABGD website (http://wwwabi.snv.jussieu.fr/public/abgd/abgdweb.html) and used the 674 CYT B sequences as input data (Table 2). We selected the K80 model and set the transition/transversion ratio at 4.73, as estimated using MEGA 7 [20]. We set both the number of steps and bins to 25 and ran the program with different gap widths as $0.5,1.0,1.5,2.0$ and 2.5. Genetic distances of $C Y T B$ between candidate species were calculated using MEGA 7 [20] under the K2P model.

We inferred the species tree using the "BEAST model [56] implemented in BEAST v. 1.8.2. Species with at least one sequence per gene were included (as required by the model). We assigned the full data set of 147 individuals to 18 candidate species groups according to the results of ABGD species delimitation analyses (Additional file 1: Table S1). We run the analyses in BEAST $\mathrm{v}$. 1.8.2 [48] for 400 million generations with the same priors as in the BEAST analyses.

To validate our ABGD proposal, a multilocus coalescent species delimitation method, BPP, was performed. Based on the phylogeny, ancestral population size, and root age, BPP employed the Reversible-Jump Markov Chain Monte Carlo (rjMCMC) method to generate the 
posterior probability that the two groups form a single species. Two data sets were used in these analyses: i) mitochondrial and nuclear genes and ii) three nuclear genes alone. Individuals represented only by mitochondrial sequences (sequences downloaded from GenBank) were not included in our BPP analyses. Using the results of AGBD, we assigned 147 individuals to 18 candidate species (Additional file 1: Table S1). We ran BPP for each of the four major clades recovered in our phylogenetic analyses separately (see Results), and used the *BEAST coalescent species tree as the guide tree. We performed the analyses using both rjMCMC algorithms 0 and 1 . Analyses were run for one million generations with a pre-burn-in of 10,000 generations and a sampling interval of 100 . We repeated BPP analyses 12 times with different combinations of priors and parameters following Wan et al. [25].

\section{Additional files}

Additional file 1: Table S1. Niviventer specimens collected for the present study. (XLSX 28 kb)

Additional file 2:Table S2. Sequence information downloaded from GenBank and used as outgroups. (XLSX 60 kb)

Additional file 3: Table S3. The best-fit partitioning schemes and evolutionary models estimated using PartitionFinder. (DOCX 17 kb)

Additional file 4: Table S4. Results of K2P genetic distance of CYT B between groups. (XLS $37 \mathrm{~kb}$ )

Additional file 5: Table S5. Algorithms, priors, and parameters for all Bayesian phylogenetics and phylogeography (BPP) analyses and results. (XLSX $16 \mathrm{~kb})$

Additional file 6: Table S6. Original description and a key to currently recognized species distributed in China. (DOCX 28 kb)

Additional file 7: Table S7. Morphological descriptions of each mitochondrial clade. (DOCX $56 \mathrm{~kb}$ )

\section{Acknowledgements}

We thank Mr. Changzhe Pu for collecting specimens.

\section{Funding}

This research was funded by the National Natural Science Foundation of China (No. 31272276) and the Fund of State Key Laboratory of Genetic Resources and Evolution, Kunming Institute of Zoology, Chinese Academy of Sciences (GREKF13-07)

\section{Availability of data and materia}

All newly conducted DNA sequences were submitted to GenBank with accession numbers KY068361-KY069019. Accession numbers of each gene locus for all specimens are also provided in Additional file 1: Table S1 and Additional file 2: Table S2.

\section{Authors' contributions}

$\mathrm{BZ}, \mathrm{KH}$, and TW contributed to the laboratory work, data analyses, and manuscript writing. PC, GZS, SYL, TSN, and LKL conducted field work and collected specimens. $X \sqcup$ conceived the study and commented on the manuscript. All authors have read and approved the final manuscript.

\section{Competing interests}

The authors declare that they have no competing interests.

\section{Consent for publication}

Not applicable.

\section{Ethics approval}

The specimen collections were approved by the internal review board of Kunming Institute of Zoology, Chinese Academy of Sciences (approval ID: SYDW-20160606001).

\section{Author details}

${ }^{1}$ State Key Laboratory of Genetic Resources and Evolution, Kunming Institute of Zoology, Chinese Academy of Sciences, Kunming, Yunnan, China. ${ }^{2}$ Kunming College of Life Science, University of Chinese Academy of Sciences, Kunming, Yunnan, China. ${ }^{3}$ National Museum of Natural History, Smithsonian Institution, Washington D.C., USA. ${ }^{4}$ Chengdu Research Base of Giant Panda Breeding, Sichuan Key Laboratory of Conservation Biology for Endangered Wildlife, Chengdu, Sichuan, China. ${ }^{5}$ China Forest Exploration and Design Institute on Kunming, Kunming, Yunnan, China. ${ }^{6}$ Sichuan Academy of Forestry, Chengdu, Sichuan, China. 'Department of Vertebrate Zoology Institute of Ecology and Biological Resources, Vietnam Academy of Sciences and Technology, Hanoi, Vietnam. ${ }^{8}$ Department of Life Sciences, Laboratory of Wildlife Ecology, Tunghai University, Taichung, Taiwan. ${ }^{9}$ The Kyoto University Museum, Kyoto University, Kyoto 606-8501, Japan.

Received: 8 June 2016 Accepted: 17 November 2016

Published online: 01 December 2016

\section{References}

1. Reeder DAM, Helgen KM, Wilson DE. Global trends and biases in new mammal species discoveries. Occasional papers The Museum of Texas Tech University. 2007;269:1-35.

2. Wilson DE, Reeder DM. Mammal species of the world. A taxonomic and geographic reference (3rd ed). Baltimore: Johns Hopkins University Press; 2005.

3. Musser GG. Notes on systematics of Indo-Malayan murid rodents, and descriptions of new genera and species from Ceylon, Sulawesi, and the Philippines. Bull Am Mus Nat Hist. 1981;168:229-334.

4. Richharia M, Westbrook LD. Walker's mammals of the world. Baltimore: Johns Hopkins University Press; 1983.

5. Allen GM. The mammals of China and Mongolia, vol. 6. New York: The American Museum of Natural History; 1940.

6. Marshall JT. Family Muridae Rats and Mice. In: Lekagul B, McNeely BA, editors. Mammals of Thailand. Thailand: Association for the Conservation of Wildlife; 1977. p. 397-485.

7. Musser GG. Species-limits of Rattus cremoriventer and Rattus langbianis, Murid Rodents of Southeast Asia and the Greater Sunda Islands. Am Mus Novit. 1973;2525:1-65.

8. Hollister N. The generic names Epimys and Rattus. Proc Biol Soc Washington. 1916:29:126.

9. Musser GG, Carleton MD. Superfamily Muroidea. In: Wilson DE, Reeder DM, editors. Mammal species of the world: a taxonomic and geographic reference (3rd ed), vol. 2. Baltimore: Johns Hopkins University Press; 2005. p. 894-1531

10. Li Y, Wu Y, Harada M, Lin LK, Motokawa M. Karyotypes of three rat species (Mammalia: Rodentia: Muridae) from Hainan Island, China, and the valid specific status of Niviventer lotipes. Zoolog Sci. 2008:25:686-92.

11. Balakirev AE, Abramov AV, Rozhnov W. Taxonomic revision of Niviventer (Rodentia, Muridae) from Vietnam: A morphological and molecular approach. Russian J Theriol. 2011;10:1-26.

12. Balakirev AE, Abramov AV, Rozhnov W. Phylogenetic relationships in the Niviventer-Chiromyscus complex (Rodentia, Muridae) inferred from molecular data, with description of a new species. Zookeys. 2014;451:109-36.

13. He K, Jiang XL. Mitochondrial phylogeny reveals cryptic genetic diversity in the genus Niviventer (Rodentia, Muroidea). Mitochondrial DNA. 2015;26(1):48-55.

14. Lu L, Ge D, Chesters D, Ho SYW, Ma Y, Li G, Wen Z, Wu Y, Wang J, Xia L, et al. Molecular phylogeny and the underestimated species diversity of the endemic white-bellied rat (Rodentia: Muridae:Niviventer) in Southeast Asia and China. Zool Scr. 2015;44:475-94.

15. Jing $M$, Yu H-T, Wu S-H, Wang W, Zheng X. Phylogenetic relationships in genus Niviventer (Rodentia: Muridae) in China inferred from complete mitochondrial cytochrome b gene. Mol Phylogenet Evol. 2007:44:521-9.

16. Balakirev AE, Rozhnov W. Phylogenic relationships and species composition in the genus Niviventer (Rodentia, Muridae) based on studies of the cytochrome b gene of mtDNA. Mosc Univ Biol Sci Bull. 2010;65:170-3. 
17. He K, Jiang XL. Sky islands of southwest China. I: an overview of phylogeographic patterns. Chin Sci Bull. 2014;59:585-97.

18. Pagés $M$, Chaval $Y$, Herbreteau $V$, Waengsothorn S, Cosson J-F, Hugot J-P, Morand S, Michaux J. Revisiting the taxonomy of the Rattini tribe: A phylogeny-based delimitation of species boundaries. BMC Evol Biol. 2010. doi:10.1186/1471-2148-10-184.

19. Lanfear R, Calcott B, Ho SYW, Guindon S. PartitionFinder: combined selection of partitioning schemes and substitution models for phylogenetic analyses. Mol Biol Evol. 2012;29:1695-701.

20. Kumar S, Stecher G, Tamura K. MEGA7: Molecular evolutionary genetics analysis version 7.0 for bigger datasets. Mol Biol Evol. 2016;33:1870-4.

21. Puillandre N, Lambert A, Brouillet S, Achaz G. ABGD, Automatic Barcode Gap Discovery for primary species delimitation. Mol Ecol. 2012;21:1864-77.

22. Confucius, Legge, J. Confucian analects, the Great learning, the Doctrine of the mean, and the Works of Mencius with English translation and notes. Montana: Kessinger Publishing; 2006.

23. Milne-Edwards H. Nouvelles Archives du Museum D'Histoire Naturelle De Paris. 1871.

24. He K, Shinohara A, Jiang X-L, Campbell KL. Multilocus phylogeny of talpine moles (Talpini, Talpidae, Eulipotyphla) and its implications for systematics. Mol Phylogenet Evol. 2014;70:513-21.

25. Wan T, He K, Jiang X-L. Multilocus phylogeny and cryptic diversity in Asian shrew-like moles (Uropsilus, Talpidae): implications for taxonomy and conservation. BMC Evol Biol. 2013;13:232.

26. Salerno PE, Senaris JC, Rojas-Runjaic FJM, Cannatella DC. Recent evolutionary history of Lost World endemics: population genetics, species delimitation, and phylogeography of sky-island treefrogs. Mol Phylogenet Evol. 2015;82:314-23.

27. Zhou W-W, Wen Y, Fu J, Xu Y-B, Jin J-Q, Ding L, Min M-S, Che J, Zhang Y-P. Speciation in the Rana chensinensis species complex and its relationship to the uplift of the Qinghai-Tibetan Plateau. Mol Ecol. 2012:21:960-73.

28. Leache AD, Fujita MK. Bayesian species delimitation in West African forest geckos (Hemidactylus fasciatus). Proc Biol Sci. 2010;277:3071-7.

29. Brown RM, Siler CD, Grismer LL, Das I, McGuire JA. Phylogeny and cryptic diversification in Southeast Asian flying geckos. Mol Phylogenet Evol. 2012; 65:351-61.

30. Rannala B. The art and science of species delimitation. Curr Zool. 2015;61:846-53.

31. ICZN. International Code of Zoological Nomenclature (4th ed). London: International Trust for Zoological Nomenclature; 1999.

32. Thomas O. On the Rat known as Epimys jerdoni from Upper Burma. J Bomb Nat Hist Soc. 1916;24:643-4.

33. Ellerman JR, Morrison-Scott TCS. Checklist of Palaearctic and Indian mammals, 1758 to 1946. London: British Museum (Natural History); 1951.

34. Feng ZJ, Cai GQ, Zheng CL. The mammals of Xizang. Beijing: Science Press; 1986.

35. Allen GM. Rats (genus Rattus) from the Asiatic Expeditions. Am Mus Novit. 1926:217:1-16

36. Ellerman JR, Hayman RW, Holt GWC, British M. The families and genera of living rodents, vol. 3. London: British museum (Natural History); 1949.

37. Sambrook J, Russell D. Molecular cloning: A laboratory manual. New York: Cold Spring Harbor Laboratory Press; 2001.

38. He K, Li Y, Brandley M, Lin L, Wang Y, Zhang Y, Jiang X. A multi-locus phylogeny of Nectogalini shrews and influences of the paleoclimate on speciation and evolution. Mol Phylogenet Evol. 2010;56:734-46.

39. Stanhope MJ, Czelusniak J, Si J-S, Nickerson J, Goodman M. A molecular perspective on mammalian evolution from the gene encoding interphotoreceptor retinoid binding protein, with convincing evidence for bat monophyly. Mol Phylogenet Evol. 1992;1:148-60.

40. Steppan SJ, Storz BL, Hoffmann RS. Nuclear DNA phylogeny of the squirrels (Mammalia: Rodentia) and the evolution of arboreality from c-myc and RAG1. Mol Phylogenet Evol. 2004;30:703-19.

41. Edgar RC. MUSCLE: multiple sequence alignment with high accuracy and high throughput. Nucleic Acids Res. 2004;32:1792-7.

42. Luo A, Qiao H, Zhang Y, Shi W, Ho SY, Xu W, Zhang A, Zhu C. Performance of criteria for selecting evolutionary models in phylogenetics: A comprehensive study based on simulated datasets. BMC Evol Biol. 2010. doi:10.1186/1471-2148-10-242

43. Fang $X$, Nevo E, Han L, Levanon EY, Zhao J, Avivi A, Larkin D, Jiang $X$ Feranchuk S, Zhu Y, et al. Genome-wide adaptive complexes to underground stresses in blind mole rats Spalax. Nat Commun. 2014. doi:10. 1038/ncomms4966.
44. Stamatakis A. RAxML version 8: a tool for phylogenetic analysis and postanalysis of large phylogenies. Bioinformatics. 2014;30:1312-3.

45. Darriba D, Taboada GL, Doallo R, Posada D. jModelTest 2: more models, new heuristics and parallel computing. Nat Methods. 2012;9:772.

46. Miller MA, Pfeiffer W, Schwartz T. Creating the CIPRES Science Gateway for inference of large phylogenetic trees. In: Gateway Computing Environments Workshop (GCE). IEEE. 2010;14:1-8.

47. Ronquist F, Teslenko M, van der Mark P, Ayres DL, Darling A, Höhna S, Larget B, Liu L, Suchard MA, Huelsenbeck JP. MrBayes 3.2: efficient bayesian phylogenetic inference and model choice across a large model space. Syst Biol. 2012;61:539-42

48. Drummond AJ, Suchard MA, Xie D, Rambaut A. Bayesian phylogenetics with BEAUti and the BEAST 1.7. Mol Biol Evol. 2012:29:1969-73.

49. Gernhard T. The conditioned reconstructed process. J Theor Biol. 2008;253:769-78.

50. Hillis DM, Bull JJ. An empirical test of bootstrapping as a method for assessing confidence in phylogenetic analysis. Syst Biol. 1993:42:182-92.

51. Huelsenbeck JP, Rannala B. Frequentist properties of Bayesian posterior probabilities of phylogenetic trees under simple and complex substitution models. Syst Biol. 2004:53:904-13.

52. Rannala B, Yang ZH. Bayes estimation of species divergence times and ancestral population sizes using DNA sequences from multiple loci. Genetics. 2003;164:1645-56.

53. Zhang J, Kapli P, Pavlidis P, Stamatakis A. A general species delimitation method with applications to phylogenetic placements. Bioinformatics. 2013:29:2869-76.

54. Fujisawa T, Barraclough TG. Delimiting species using single-locus data and the generalized mixed yule coalescent approach: a revised method and evaluation on simulated data sets. Syst Biol. 2013;62:707-24

55. Schwarzfeld MD, Sperling FA. Comparison of five methods for delimitating species in Ophion Fabricius, a diverse genus of parasitoid wasps (Hymenoptera, Ichneumonidae). Mol Phylogenet Evol. 2015;93:234-48.

56. Heled J, Drummond AJ. Bayesian inference of species trees from multilocus data. Mol Biol Evol. 2010;27:570-80

\section{Submit your next manuscript to BioMed Central and we will help you at every step:}

- We accept pre-submission inquiries

- Our selector tool helps you to find the most relevant journal

- We provide round the clock customer support

- Convenient online submission

- Thorough peer review

- Inclusion in PubMed and all major indexing services

- Maximum visibility for your research

Submit your manuscript at www.biomedcentral.com/submit 